\title{
Power Consumption and Temperature Distribution in WRGB Active-Matrix OLED Displays
}

\author{
Frédérique Chesterman, Grigorij Muliuk, Bastian Piepers, Tom Kimpe, Patrick De Visschere, and Kristiaan Neyts
}

\begin{abstract}
In this paper, the power consumption of a white-red-green-blue (WRGB) active-matrix organic light-emitting device (OLED) display and the resulting temperature distribution across the display are analyzed as a function of the applied image and the luminance of the emitted light. It has been shown previously that temperature directly impacts the picture quality of an OLED display. Luminance, spectral radiance, power and temperature measurements are performed on a 55-in WRGB OLED display with a resolution of $1920 \times 1080$. A power model is presented that allows calculating the display's power consumption for a given applied image. This involves the dependency of the efficiency of the white OLED on the current density, the wavelength dependent transmission of the color filters and the contribution of each of the subpixels in producing the display's nominal white. The output of the power model is used as input for a basic thermal model that simulates the temperature distribution across the display. The thermal model is based on 3D computational fluid dynamics analysis framework (FloEFD). A good agreement between the simulations and measurements on the sample WRGB OLED display is obtained.
\end{abstract}

Index Terms-Filter characteristics, OLED efficiency, power consumption, temperature distribution, WRGB OLED display.

\section{INTRODUCTION}

$\mathbf{T}$ WENTY-EIGHT years have passed since the discovery of the organic light-emitting device (OLED) working principle [1]. Today, OLED technology has become ubiquitous, with applications in many different display markets and mass production of both small- and large-sized OLED displays. However, for demanding display applications, such as medical imaging, the performance of OLED technology is currently not yet meeting the stringent requirements [2], [3]. One of the reasons for this is the difficulty of an OLED display to maintain constant picture quality. Phenomena responsible for this are the

Manuscript received November 20, 2015; accepted December 22, 2015. Date of publication December 31, 2015; date of current version May 16, 2016. This work was supported by the IWT (Institute for the Promotion of Innovation by Science and Technology in Flanders) in the context of the Baekeland Grant "Applicability of OLED technology in medical displays" under Grant 130300.

F. Chesterman, B. Piepers, and T. Kimpe are with the Healthcare Division, Barco NV, Kortrijk 8500, West-Vlaanderen, Belgium (e-mail: frederique.chesterman@barco.com; bastian.piepers@barco.com; tom. kimpe@barco.com).

G. Muliuk is with Ghent University, INTEC, Gent B-9000, Belgium (e-mail: grigorij.muliuk@ugent.be).

P. De Visschere and K. Neyts are with Ghent University, ELIS, Gent B-9000, Belgium (e-mail:pdv@elis.ugent.be; kneyts@elis.ugent.be).

Color versions of one or more of the figures are available online at http:// ieeexplore.ieee.org.

Digital Object Identifier 10.1109/JDT.2015.2513819 temperature dependency of the OLED's light output [4]-[7], and OLED aging, for which the rate is temperature dependent [8], [9].

It is clear from the above description that the influence of temperature on the performance of an OLED display cannot be neglected. A detailed understanding of the parameters that determine the temperature of an OLED display and its distribution is essential as a step towards reliably performing OLED displays. In response to this need, this paper investigates the power consumption of an OLED display as a function of the applied image and the resulting temperature distribution across the display.

The focus in this paper is on white-red-green-blue (WRGB) OLED display technology which is introduced in Section II. Measurements performed on a sample 55-in WRGB OLED display with a resolution of $1920 \times 1080$ are discussed in Section III. In Section IV, a model is presented that allows simulating the temperature distribution across the display for a given applied image. Determination of the temperature distribution occurs in a two-step process. First, the display's power consumption is calculated for a given luminance profile by means of a WRGB OLED display power model (cf. Section IV-A). Analytic expressions for the relation between power consumption and luminance are derived. The calculated power consumption is used as input for a thermal model (cf. Section IV-B). This model is based on a 3D computational fluid dynamics analysis framework (FloEFD) and consists of a computer-aided design (CAD) drawing of a WRGB OLED display with dimensions and materials similar to the display under test. The thermal model is meant to be generic for OLED displays. This means we limit the components in the model to what is typical for such displays.

Although both the power model and the thermal model can simulate power consumption and temperature distribution for random image content, we will limit the study in this article to full-screen uniform images at different Digital Driving Levels (DDL). The output of both the power model and the thermal model are verified by means of measurements on the WRGB OLED display.

\section{WRGB OLED DisPLAY TECHNOLOGY}

WRGB OLED technology is currently the OLED technology that is most mature for large-size OLED displays. Each pixel in a WRGB OLED display comprises four subpixels that are based on white OLEDs. A full color display is obtained by placing color filters in front of three subpixels, as shown schematically 


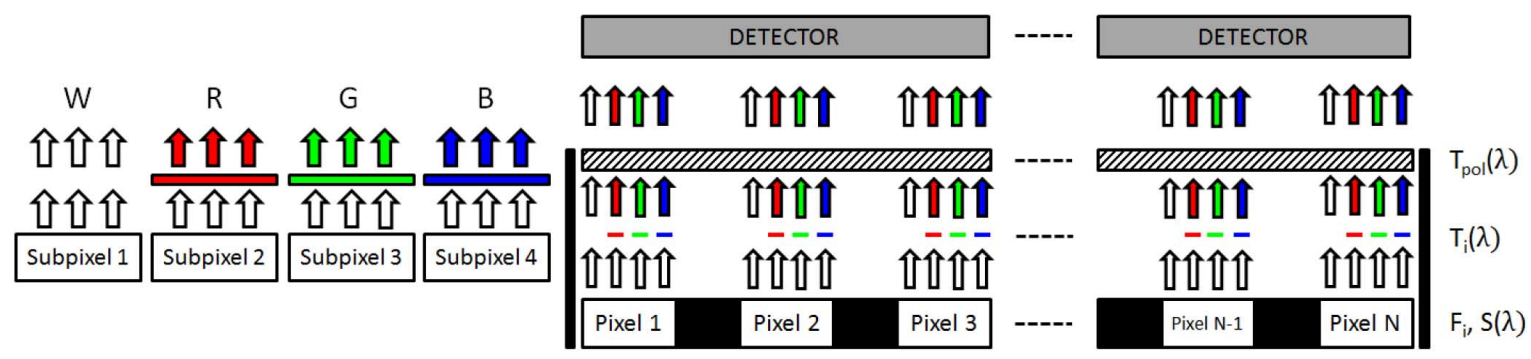

(a) Pixel structure

(b) Panel structure

Fig. 1. (a) Pixel structure of a WRGB OLED display. Each pixel comprises four subpixels. Three of them have a color filter placed in front of them to convert the white light into colored light. (b) \{anel structure. Layers in a WRGB OLED display. The display is composed of a matrix of pixels. Each pixel contains four subpixels, each of them emitting white light. Three subpixels have a color filter placed in front of them to convert the white light into colored light. The filtered light subsequently passes a polarizer and front glass. A light measurement device measures an average of the light across the surface of the display.

in Fig. 1(a). A fourth subpixel has no filter in order to increase the white efficiency.

To obtain the desired white point for the display, the light emitted from the white subpixel is supplemented with light from the blue and red subpixel (cf. Section III-A1). This means that DDLs of the form $\left(D D L_{R}, D D L_{G}, D D L_{B}\right)$ with $D D L_{R}=$ $D D L_{G}=D D L_{B}$ do not allow investigating the behavior of the white subpixel separately. However, as the quadruplet $(W, R, G, B)$ representing the driving of each of the four subpixels is a linear transformation of the DDL triplet $\left(D D L_{R}\right.$, $\left.D D L_{G}, D D L_{B}\right)$, there must exist one triplet for which only the white subpixel is used. This triplet was determined experimentally as $(252,255,215)$. In the remainder of this article we will refer to "the display's nominal white" and "the light emitted from the white subpixel" to indicate the light that is obtained when driving the display at a DDL of the form $\left(D D L_{R}, D D L_{G}\right.$, $D D L_{B}$ ) with $D D L_{R}=D D L_{G}=D D L_{B}$ and $D D L$ between 1 and 255 and DDL $(252,255,215)$ respectively.

\section{Measurements}

In this section, the results of a number of different measurements on the 55-in WRGB OLED display will be presented. In Section IV, these measurements will be used to construct the models.

\section{A. Luminance and Power}

The power consumption of a 55-in WRGB OLED display with a resolution of $1920 \times 1080$ is investigated as a function of the luminance level. All power measurements are executed using a Chroma 66200 Digital Power Meter. All luminance measurements are executed using a Konica Minolta CA-210 Display Color Analyzer. The measurement head of the Konica Minolta CA-210 is placed perpendicular to the display surface such that luminance is measured in perpendicular direction. The power consumption and luminance are measured for uniform full-screen patches (all pixels active and driven in the same way) at different DDLs: (DDL, 0,0$),(0, \mathrm{DDL}, 0),(0,0, \mathrm{DDL})$ and (DDL,DDL,DDL), respectively red, green, blue and nominal white, with DDL ranging from 45 to 255 with step-size 15 . Measurement results are shown in Fig. 2 for luminance and Fig. 3 for power consumption.

The measurements show that the display consumes a significant amount of power at DDL $(0,0,0)$ (approximately $42 \mathrm{~W}$ ).

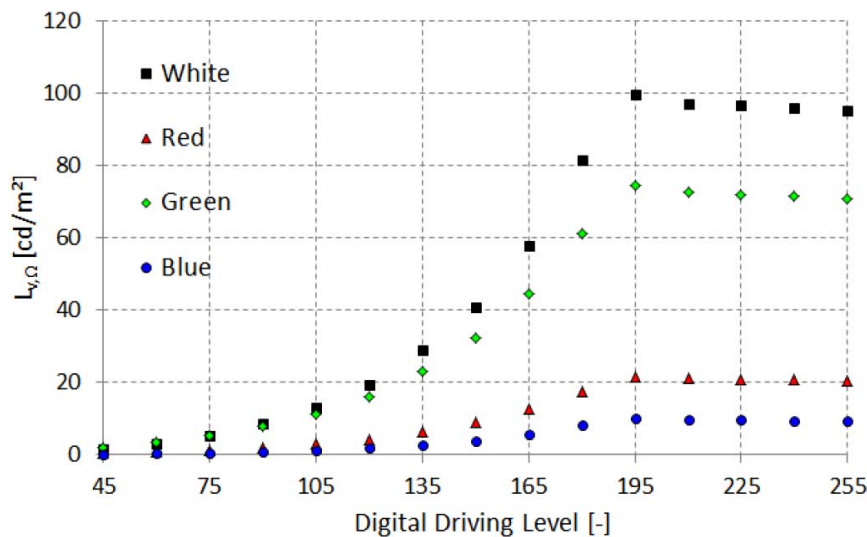

Fig. 2. Measured luminance $L_{e, \Omega}$ as a function of Digital Driving Level (DDL) for red (DDL, 0,0$)$, green $(0, \mathrm{DDL}, 0)$, blue $(0,0, \mathrm{DDL})$, and nominal white (DDL,DDL,DDL).

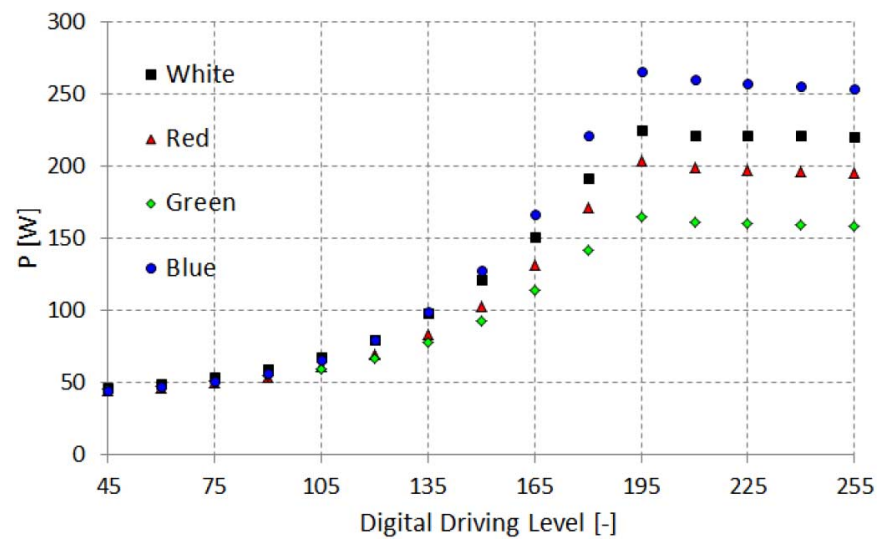

Fig. 3. Measured power consumption $P$ as a function of Digital Driving Level (DDL) for red (DDL, 0,0$)$, green $(0, \mathrm{DDL}, 0)$, blue $(0,0, \mathrm{DDL})$, and nominal white (DDL,DDL,DDL).

This non-zero power consumption can partially be attributed to losses in the power lines at the location where the power is supplied to the display. Indeed, at DDL $(0,0,0)$, we observe zones at the bottom of the display with temperature more than five degrees above ambient (cf. Section III-C). Another part of the power consumption can be attributed to the power consumption of the electronic boards that process the data signal. (cf. Section III-C). 


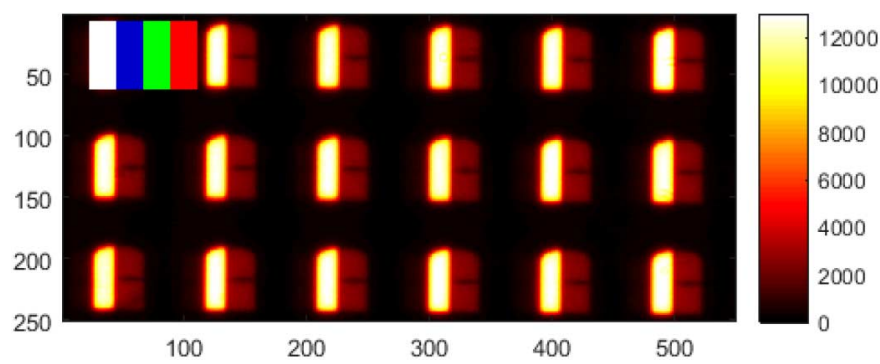

Fig. 4. Close-up CCD camera capture of 18 pixels $(4 \times 18$ subpixels $)$ in a WRGB OLED display when the pixels are driven at DDL $(255,255,255)$. The white, blue and red subpixels are active. The intensity of the red subpixel is so low that it is barely visible on the image. Colored rectangles are added in the top-left corner of the image to indicate the location of the white, red, green and blue subpixel. The CCD camera is a grayscale device. The captured image is a matrix of intensity values with one value for each of the pixels in the camera's sensor. The intensity is determined by the power and wavelength of the emitted light and the spectral response of the sensor.

The measurements further show that the power consumption is the largest for blue, followed by nominal white, red and green in decreasing order. Finally, both luminance and power consumption saturate for DDLs above 195. This is a typical OLED display behavior and is a deliberate design parameter of display manufacturers to avoid excessive power losses in the driving electronics and associated heat creation in the display.

1) Contribution of Each of the Sub-Pixels in Producing the Display's Nominal White: In interpreting the power consumption for DDLs of the form (DDL,DDL,DDL), i.e. the display's nominal white, it should be noted that the display not only uses the white subpixel, but also the blue subpixel and the red subpixel (cf. Section II). A cooled charge-coupled device (CCD) camera is used to capture a close-up picture of the pixel structure when showing a nominal white uniform full-screen patch. The result is shown in Fig. 4. Analysis of the close-up image indicates that the light output of the blue pixel when showing DDL $(255,255,255)$ is approximately $55.3 \%$ of the light output from the blue subpixel when the display is driven at DDL $(0,0,255)$. When showing DDL $(255,255,255)$, the light output from the red subpixel is approximately $2.7 \%$ of the light output when showing DDL $(255,0,0)$. This means that, of the $95.34 \mathrm{~cd} / \mathrm{m}^{2}$ produced by the display when showing DDL $(255,255,255)$, approximately $5.19 \mathrm{~cd} / \mathrm{m}^{2}$ is delivered by the blue subpixel, $0.55 \mathrm{~cd} / \mathrm{m}^{2}$ by the red subpixel and the remaining $89.61 \mathrm{~cd} / \mathrm{m}^{2}$ by the white subpixel.

\section{B. Spectral Radiance}

The spectral radiance of the light emitted by each of the subpixels is measured by means of a Yeti Specbos spectrometer. To that extent, uniform full-screen patches with the three color primaries as well as the DDL for which only the white subpixel is used (DDL $(252,255,215))$ are sequentially applied to the display. The DDLs for the color primaries were chosen such that the display's power consumption is very close to the power consumption at DDL $(252,255,215)(103.2 \mathrm{~W})$. Those DDLs are $(149,0,0)(102.4 \mathrm{~W}),(0,157,0)(102.6 \mathrm{~W})$ and $(0,0,136)$ $(102.5 \mathrm{~W})$. The four measured spectra are shown in Fig. 5.

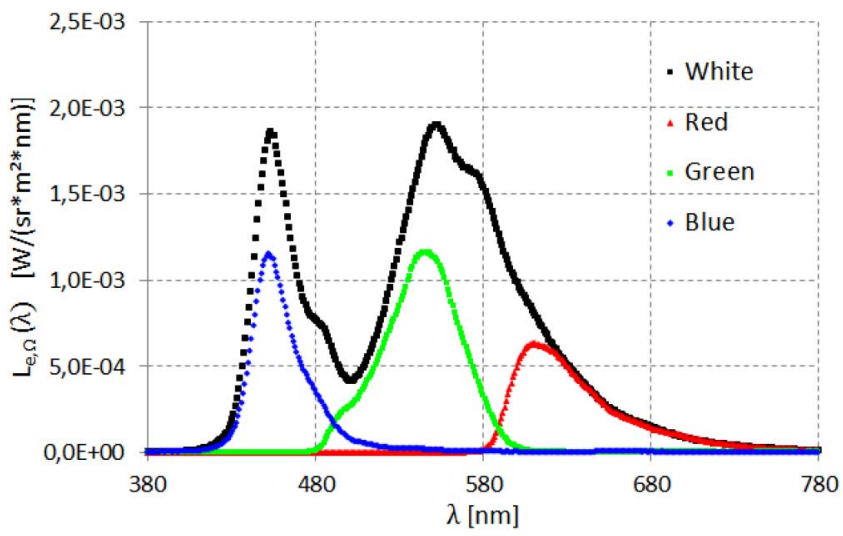

Fig. 5. Measured spectral radiance $L_{e, \Omega}(\lambda)$ for red, green, blue, and white. For each color, the DDL was chosen such that the power consumption $P$ of the display for each color is equal.
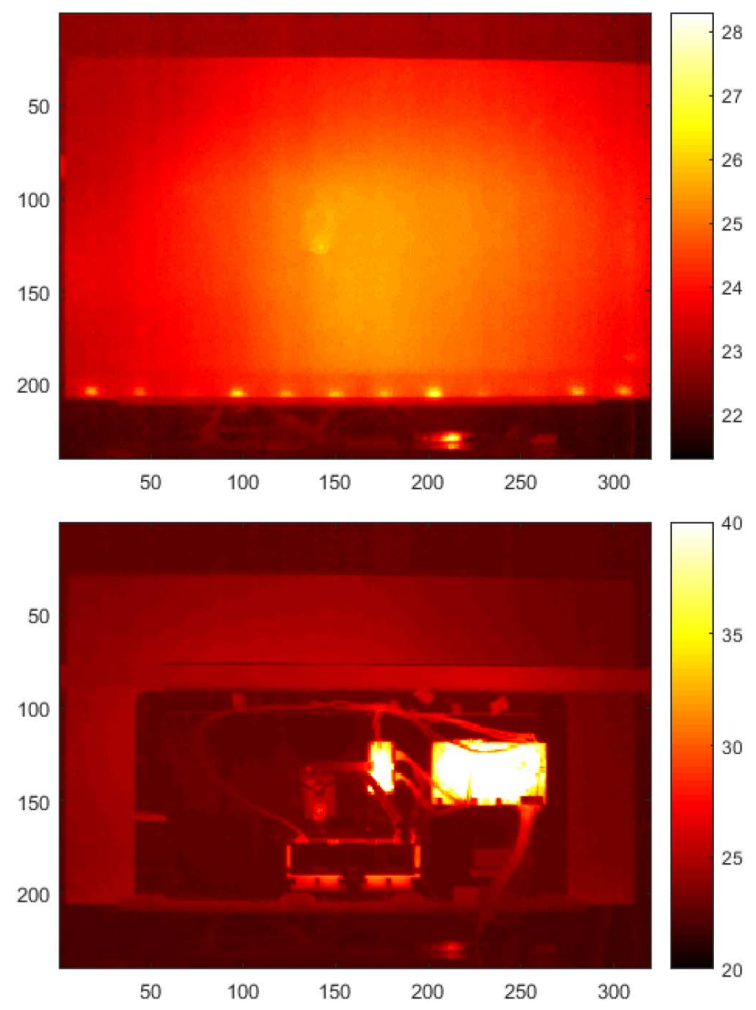

Fig. 6. IR camera image of the WRGB OLED display when a uniform fullscreen black image (DDL $(0,0,0))$ is displayed: front-view (top) and rear-view (bottom). On the front-view image one can notice spots at the bottom of the display at a temperature of five degrees above ambient. On the rear-view image, one can distinguish between the hot electronic boards at the back of the display, a center part of the display with low radiation and an outer part of the display with high radiation.

\section{Temperature Distribution}

The significant power consumption in a WRGB OLED display causes an elevated temperature distribution across the display [10]-[12]. Experiments were set up to investigate the maximum temperature and temperature distribution across the display for uniform full-screen patches at different DDLs.

The temperature distribution of the display is captured using an infrared (IR) camera with resolution of $320 \times 240$ pixels. The emissivity coefficient of the camera was set to 1 , which means 

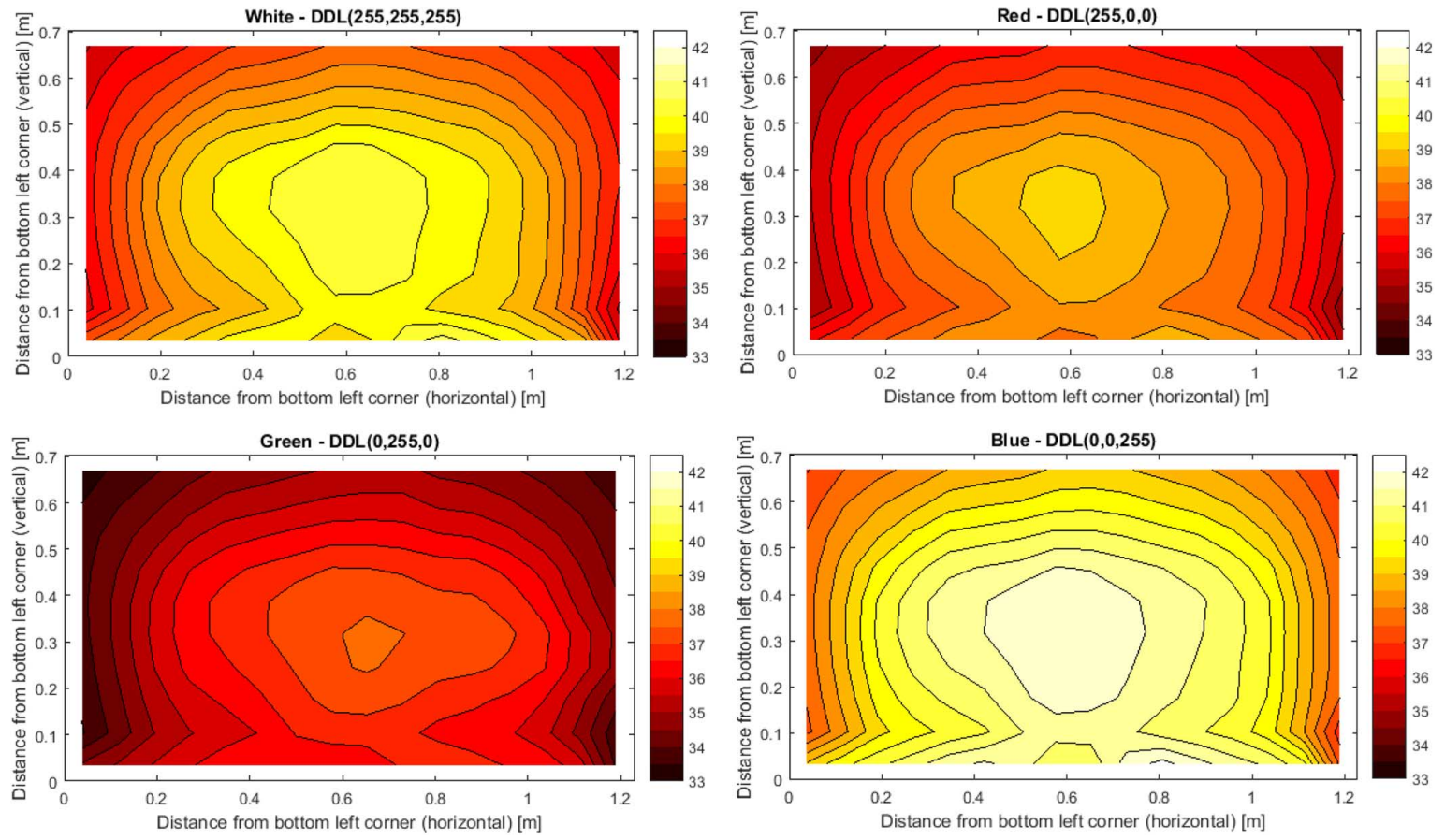

Fig. 7. Measured steady-state temperature distribution across the OLED display for a full-screen nominal white (top-left), red (top-right), green (bottom-left) and blue uniform full-screen patch (bottom-right). The figures show the temperature distribution across the entire display surface. The values on the $\mathrm{x}$ and $\mathrm{y}$-axes correspond to the actual display dimensions. The color bar indicates the temperature in degrees centigrade.

the IR camera expects it is measuring a perfect blackbody radiator. As the front glass of the display is not transparent to IR radiation, the IR camera measures the temperature of the outer surface of the display's front glass rather than the temperature of the OLED pixels. Between the heat source (the OLED pixels) and the measurement location (the outer surface of the glass plate) we can identify two thermal resistances. The first thermal resistance corresponds to the thermal barrier between the OLED and the glass plate. The second thermal resistance corresponds to the thermal barrier induced by the glass plate. Concerning the thermal barrier between the OLED and the glass plate, we assume the OLED to be in thermal contact with at least one side of the display. Because of the very small dimensions of the OLED (maximum a few micrometers), the thermal resistance between the OLED and the glass plate is very small, independent of the material properties. As the glass plate is thin, also the thermal resistance induced by the glass plate is small. For the blue uniform full-screen patch (power consumption $=179.9 \mathrm{~W}$ ) we calculate a temperature gradient of approximately $0.15^{\circ} \mathrm{C}$ between both sides of the glass plate assuming the heat escapes from the display both at the front and at the backside. From this analysis we can conclude that the temperature measured with the IR camera will be very close to the temperature of the OLED pixels.

Four experiments are conducted in which respectively a nominal white, red, green and blue uniform full-screen patch at maximum DDL is displayed. In all experiments, the patch is displayed on the screen for two hours prior to the measurement such that a steady state temperature distribution is reached. Ambient temperature during the measurements is $25.6{ }^{\circ} \mathrm{C}, 26^{\circ} \mathrm{C}$, $26.1^{\circ} \mathrm{C}$ and $25.8^{\circ} \mathrm{C}$ for nominal white, red, green and blue respectively. The measured power consumption of the display (as well as the luminance of the emitted light) decreased slightly compared to the values shown in Fig. 3 after the extended operating time of two hours to $213.7 \mathrm{~W}, 189.3 \mathrm{~W}, 161.7 \mathrm{~W}$ and $228.2 \mathrm{~W}$ for nominal white, red, green and blue respectively. This is believed to be caused by an internal compensation algorithm that recalibrates the display as a function of the display temperature. The power measurements include the $42 \mathrm{~W}$ of power consumed in the electronic boards at the back of the display. It can be seen from Fig. 6 that there is significant heat creation in the electronic boards at the back of the display.

During the actual measurements, the pixels are switched off during one second every 30 seconds to avoid that the display would reduce its luminance. Indeed, the WRGB OLED display under test houses a second algorithm that reduces the display's luminance when static content is displayed. The average power consumed in the OLED display itself is therefore determined as

$$
P_{\text {average }}=\left(P-P_{\text {offset }}\right) * \text { fraction }_{\text {on }}
$$

with $P_{\text {offset }}$ the power consumption when all pixels are switched off $(42 \mathrm{~W})$ and fraction $_{\text {on }}$ the fraction of the time that the pixels are switched on during the measurements (29/30). This results in an actual power consumption $P_{\text {average }}$ of $166 \mathrm{~W}$, $142.4 \mathrm{~W}, 115.7 \mathrm{~W}$ and $179.9 \mathrm{~W}$ for nominal white, red, green and blue respectively.

The measured temperature distributions for nominal white, red, green and blue are shown in Fig. 7. The maximum temperature reached on the display for nominal white, red, green and blue is shown as a function of the power consumption in Fig. 8. The maximum temperature increases linearly as a function of the power consumption. The maximum temperature 


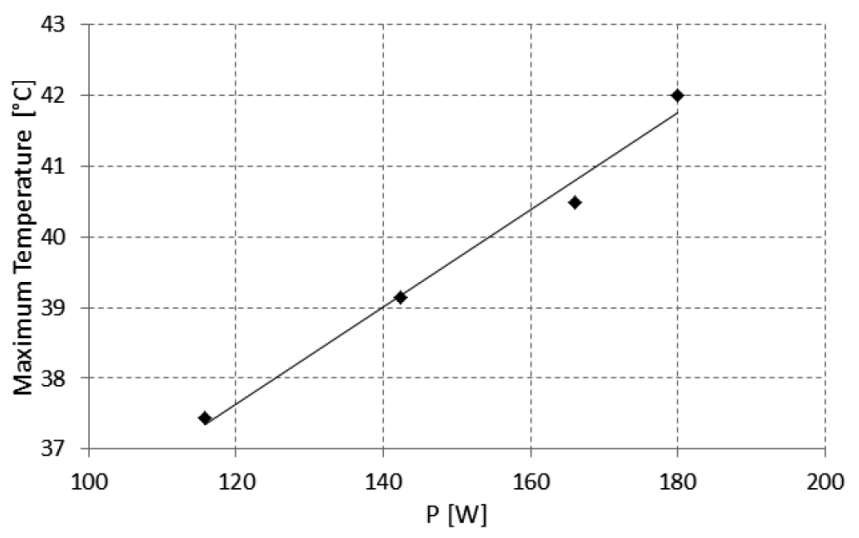

Fig. 8. Maximum temperature across the WRGB OLED display for nominal white, red, green, and blue as a function of the power consumption of the display. Uniform full-screen patches are applied for two hours continuously prior to the measurements such that a steady-state temperature distribution is reached.

is found in the center of the screen and a relatively strong temperature gradient is found across the screen. In correspondence to the increased temperature that is found at the bottom of the display when a black uniform full-screen patch is shown (cf. Fig. 6), the experiments show an increased temperature at the bottom of the display.

\section{DESCRIPTION OF THE MODEL}

In this section, we present a model that allows calculating the temperature distribution across a WRGB OLED display for a given applied image (matrix of DDLs). This model is composed of two submodels: a first model describes the relation between the image shown on the display and the power consumption of the display; a second model describes the relation between the power consumption of the display and the temperature distribution across the display. A schematic description of the model is shown in Fig. 9. The relation between the luminance of the external light emission and the DDL is assumed to be known and available in the form of a look up table. The power model and the thermal model will be treated in detail in Section IV-A and Section IV-B, respectively.

\section{A. Power Model}

1) Assumptions: In a WRGB OLED display, a voltage is applied to each column line. The current flowing through the line is determined by the DDL of each of the pixels connected to that column and equal to the sum of the currents supplied to the pixels. At any time, the total power consumed by the column is equal to the product of the maximum current flowing through the line and the column voltage. In the model presented below, it is assumed that all of this power is consumed in the OLED pixels themselves. In reality however, part of the power consumption takes place in the power lines and in the thin-film transistors (TFTs) because of the resistivity of the power line and the TFT channel respectively. This assumption does not influence the output of the power model as the model calculates the display's total power consumption. The exact location of the heat sources will however be influenced. Indeed, in the thermal model presented in Section IV-B, it is assumed that all heat sources are located on the OLED pixels themselves. In reality, a small part
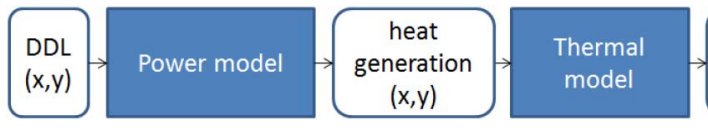

temperature distribution $(x, y, z, t)$

Fig. 9. Schematic description of the model. A power model translates an image (matrix of DDLs) into a number of heat sources across the display. A thermal model calculates the spreading of the heat across the display.

of the heat will be created in the power lines, at a different location than the OLED pixels. It is assumed that the influence of this on the temperature distribution is limited.

2) Definition: When an image is shown on the screen, a current that is a function of the luminance of the emitted light flows through each of the subpixels. As is shown schematically in Fig. 1(b), the light emitted from the white OLEDs in a WRGB OLED display passes through a color filter, a polarizer and the front glass before being emitted from the display. Radiance and luminance measured at the outside of the display are an average across pixel and non-pixel regions.

With $i$ ranging from 1 to 4 , indicating white, red, green and blue respectively, the relation between the spectral radiance of the external light emission $L_{e, \Omega, i}(\lambda)\left[W /\left(s r . m^{2}\right)\right]$ for color $i$ and the current density $J_{i}$ flowing through subpixel $i$ can be expressed as

$$
L_{e, \Omega, i}(\lambda)=\eta\left(J_{i}\right) J_{i} F_{i} S(\lambda) T_{i}(\lambda) T_{p o l}(\lambda)
$$

with $\eta\left(J_{i}\right)[W /(s r . A)]$ the efficacy of the white OLED, $J_{i}\left[A / m^{2}\right]$ the current density, $S(\lambda)[-]$ the normalized spectral dependency of the light emitted from the white subpixel with $\int S(\lambda) d \lambda=1, T_{i}(\lambda)[-]$ the wavelength dependent filter transmission of the filter in front of subpixel $i, T_{p o l}(\lambda)[-]$ the wavelength dependent transmission of the polarizer and $F_{i}[-]$ the fill factor of subpixel $i$. Consequently, the relation between the radiance of the external light emission $L_{e, \Omega, i}$ and the current density $J_{i}$ is given by

$$
L_{e, \Omega, i}=\int \eta\left(J_{i}\right) J_{i} F_{i} S(\lambda) T_{i}(\lambda) T_{p o l}(\lambda) d \lambda .
$$

The luminance of the external light emission $L_{v, \Omega, i}\left[c d / \mathrm{m}^{2}\right]$ is subsequently found as

$$
L_{v, \Omega, i}=\int L_{e, \Omega, i}(\lambda) \bar{y}(\lambda) d \lambda
$$

with $\bar{y}(\lambda)[l m / W]$ the wavelength dependent eye sensitivity.

Finally, the total power consumption $P_{\text {total }}$ of the display can be expressed as

$$
P_{\text {total }}=\sum_{i} N V_{D D} J_{i} A F_{i}+P_{o f f s e t}
$$

with $\mathrm{N}[-]$ the number of pixels in the display, $V_{D D}$ the column voltage (i.e. the total voltage across the OLED, the TFT and the power line) and $P_{\text {off set }}$ the power consumed by the display when the screen is completely black.

We replace the wavelength dependent filter transmission $T_{i}(\lambda)$, polarizer transmission $T_{p o l}(\lambda)$ and eye sensitivity $\bar{y}(\lambda)$ by the averaged scalars $\left\langle T_{i}\right\rangle,\left\langle T_{p o l}\right\rangle$ and $\langle\bar{y}\rangle$ respectively. These 
TABLE I

PARAMETERS OF THE WRGB DISPLAY

\begin{tabular}{|c||c||c||c||c|}
\hline & White & Red & Green & Blue \\
\hline $\mathrm{N} \mathrm{[-]}$ & 2073600 & 2073600 & 2073600 & 2073600 \\
\hline$V_{D D}[\mathrm{~V}]$ & 12 & 12 & 12 & 12 \\
\hline$A\left[m^{2}\right]$ & $0.391 \mathrm{e}-6$ & $0.391 \mathrm{e}-6$ & $0.391 \mathrm{e}-6$ & $0.391 \mathrm{e}-6$ \\
\hline$\left\langle T_{i}\right\rangle[-]$ & 1 & 0.178 & 0.282 & 0.171 \\
\hline$F_{i}[-]$ & 0.0879 & 0.0766 & 0.0676 & 0.1056 \\
\hline$\left\langle\bar{y}_{i}\right\rangle[-]$ & 369.8 & 208.9 & 575.0 & 68.9 \\
\hline
\end{tabular}

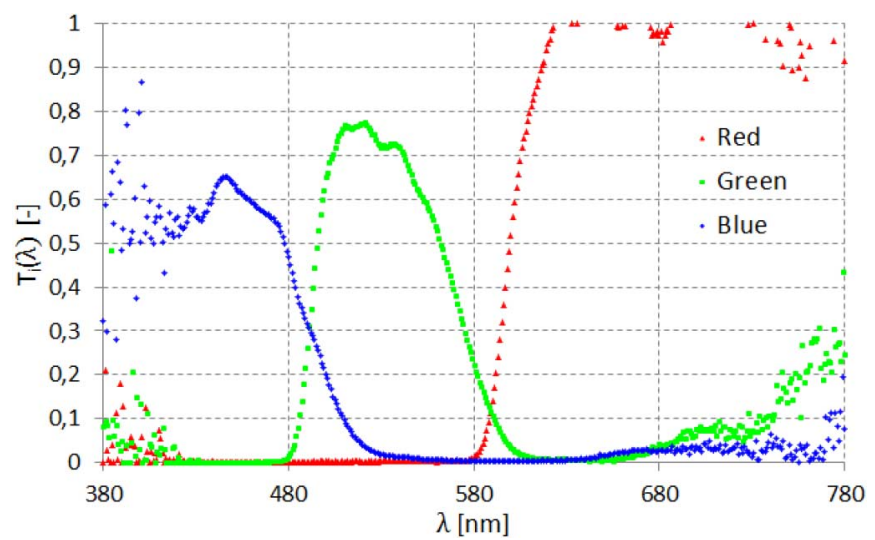

Fig. 10. Filter characteristics $T_{i}(\lambda)$ for the filter in front of the red, greens and blue subpixels.

scalars are averages of the wavelength dependent characteristics weighted with the spectrum of the white light $S(\lambda)$, according to the following expressions:

$$
\begin{aligned}
\left\langle T_{\text {pol }}\right\rangle & =\int T_{\text {pol }}(\lambda) S(\lambda) d \lambda \\
\left\langle T_{i}\right\rangle & =\frac{\int T_{i}(\lambda) S(\lambda) T_{\text {pol }}(\lambda) d \lambda}{\int S(\lambda) T_{\text {pol }}(\lambda) d \lambda} \\
\langle\bar{y}\rangle & =\frac{\int L_{e, \Omega, i}(\lambda) \bar{y}(\lambda) d \lambda}{\int L_{e, \Omega, i}(\lambda) d \lambda} .
\end{aligned}
$$

Using these expressions one can rewrite (3) and (4) as follows:

$$
\begin{aligned}
L_{e, \Omega, i} & =\eta\left(J_{i}\right) J_{i} F_{i}\left\langle T_{i}\right\rangle\left\langle T_{p o l}\right\rangle \\
L_{v, \Omega, i} & =L_{e, \Omega, i}\langle\bar{y}\rangle .
\end{aligned}
$$

These expressions can be used for calculating the current through each of the subpixels for a given luminance profile. Once all current densities have been determined, the total power consumption is obtained by means of (5).

3) Parameter Extraction: Before the model presented above can be used one needs to determine numeric values for the parameters $\left\langle T_{i}\right\rangle,\left\langle T_{\text {pol }}\right\rangle$ and $\langle\bar{y}\rangle$. The value for $\langle\bar{y}\rangle$ follows immediately from the eye sensitivity function $\bar{y}(\lambda)$. One can determine the values for $\left\langle T_{i}\right\rangle$ from spectral measurements on the display. Combining (7) and (3) for color i and for white we find

$$
\left\langle T_{i}\right\rangle=\frac{\frac{\int L_{e, \Omega, i}(\lambda) d \lambda}{\eta\left(J_{i}\right) J_{i} F_{i}}}{\frac{\int L_{e, \Omega, w}(\lambda) d \lambda}{\eta\left(J_{w}\right) J_{w} F_{w}}}
$$

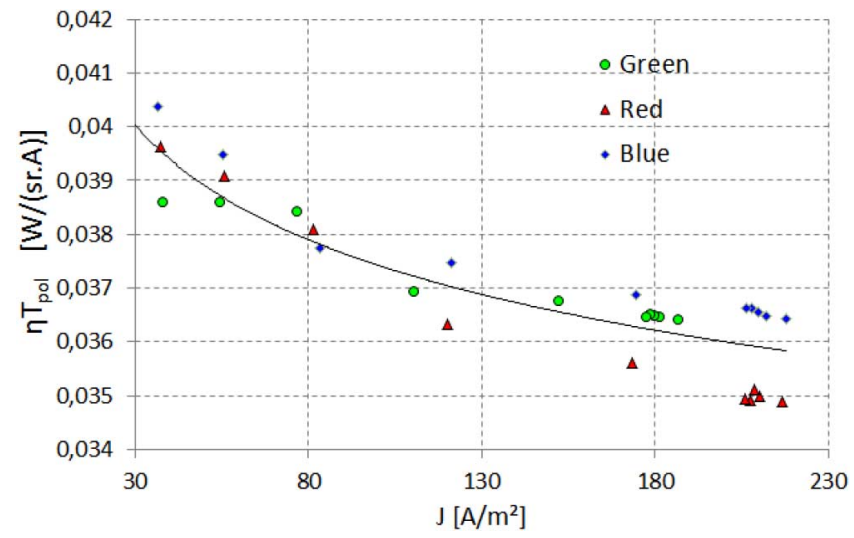

Fig. 11. Efficacy $\eta T_{p o l}$ of the white OLED as a function of current density $J$ for the red, green and blue subpixel.

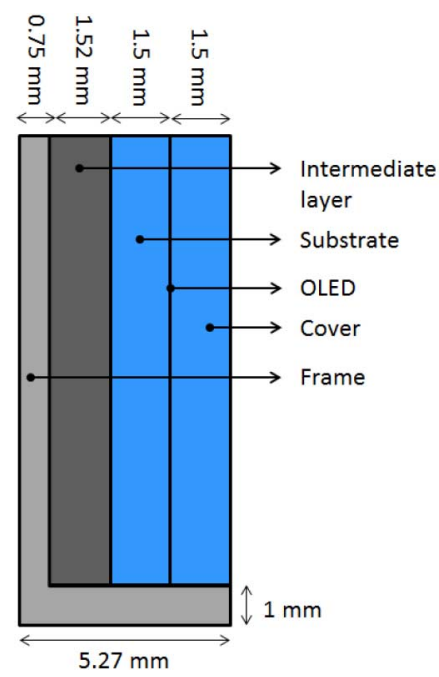

Fig. 12. Schematic drawing of the CAD model used in FloEFD for the temperature distribution simulation: Side-view. The CAD model consists of an OLED heat source sandwiched between a glass substrate and a glass cover and integrated into an aluminum frame. In between the aluminum frame and glass substrate there is an intermediate layer for which the thermal properties are tuned. The dimensions of the different components are indicated in the figure.

with $i$ referring to color $i$ and $w$ referring to the DDL for which only the white subpixel is used. For equal current densities $\left(J_{i}=\right.$ $J_{w}$ ), this expression can be simplified to:

$$
\left\langle T_{i}\right\rangle=\frac{\int L_{e, \Omega, i}(\lambda) d \lambda}{\int L_{e, \Omega, w}(\lambda) d \lambda} \frac{F_{w}}{F_{i}} .
$$

This expression is used to determine the filter transmissions $\left\langle T_{i}\right\rangle$. The first factor is the ratio of the radiance measured for color $i$ and the radiance measured for white. Similarly, the wavelength dependent transmission characteristics of the color filters can be determined as

$$
T_{i}(\lambda)=\frac{L_{e, \Omega, i}(\lambda)}{L_{e, \Omega, w}(\lambda)} \frac{F_{w}}{F_{i}} .
$$

Once the filter characteristics have been determined, one can find the product $\left\langle T_{p o l}\right\rangle \eta\left(J_{i}\right)$ from (3), (6), and (7) or immediately from (9):

$$
\eta\left(J_{i}\right)\left\langle T_{p o l}\right\rangle=\eta\left(J_{i}\right) \int S(\lambda) T_{p o l}(\lambda) d \lambda
$$




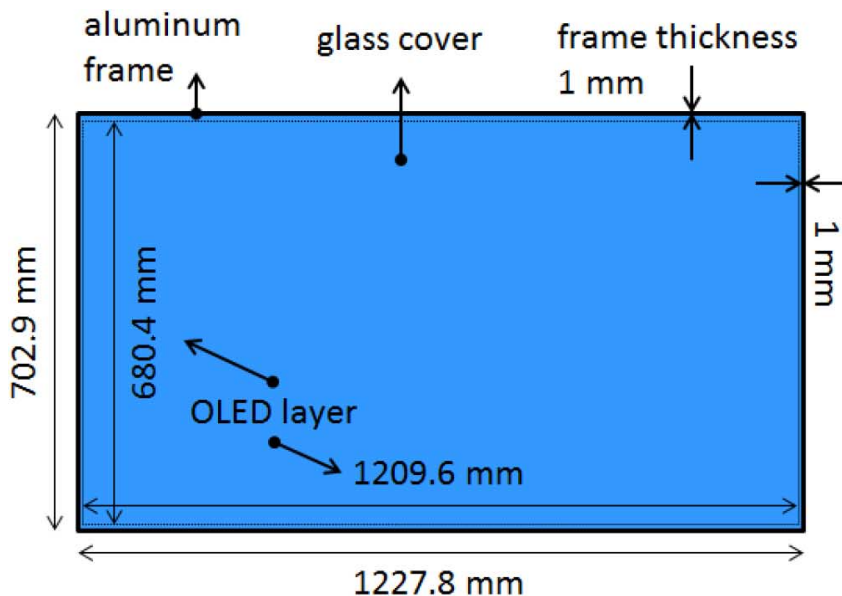

(a) Front-view

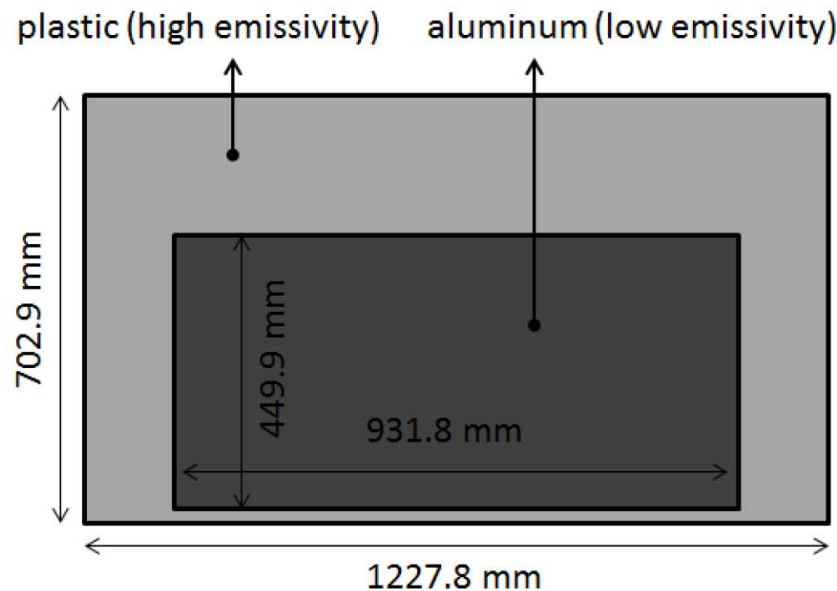

(b) Rear-view

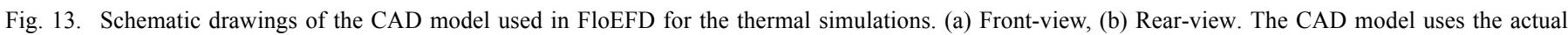

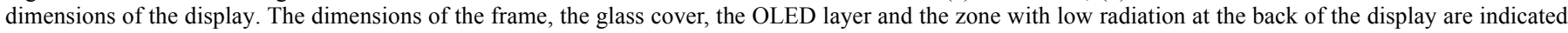
in the figure.

TABLE II

Parameters of the Materials Used in the Thermal Model

\begin{tabular}{|c||c||}
\hline Material & Thermal conductivity \\
\hline Glass & 1.05 \\
Aluminum & 237 \\
Intermediate layer & 15.1 \\
\hline
\end{tabular}

$$
\begin{aligned}
& =\eta\left(J_{i}\right) \int T_{i}(\lambda) S(\lambda) T_{\text {pol }}(\lambda) d \lambda \frac{1}{\left\langle T_{i}\right\rangle} \\
& =\frac{L_{e, \Omega, i}}{J_{i} F_{i}\left\langle T_{i}\right\rangle} .
\end{aligned}
$$

In this case, $\left\langle T_{\text {pol }}\right\rangle$ and $\eta\left(J_{i}\right)$ are not known separately, but not needed either.

Using the above formulas, values for the parameters $\left\langle T_{i}\right\rangle$, $\left\langle T_{\text {pol }}\right\rangle \eta\left(J_{i}\right)$ and $\langle\bar{y}\rangle$ have been determined from the measurements in Section III. The values for $\left\langle T_{i}\right\rangle$ and $\langle\bar{y}\rangle$ as well as some typical values for the other parameters used in Section IV are listen in Table I. The wavelength dependent filter characteristics $T_{i}(\lambda)$ are shown in Fig. 10. Finally, $\left\langle T_{p o l}\right\rangle \eta\left(J_{i}\right)$ is shown as a function of the current density in Fig. 11.

By multiplying $\left\langle T_{p o l}\right\rangle \eta\left(J_{i}\right)\left(0.040 \mathrm{~W} /(\right.$ sr.A $)$ at $\left.30 \mathrm{~A} / \mathrm{m}^{2}\right)$ with $\left\langle\bar{y}_{i}\right\rangle$ for white, we find an efficacy of $14.8 \mathrm{~cd} / \mathrm{A}$ for the white OLED and polarizer combined. We further obtain an efficiency of $1.05 \%$ [W/W] by multiplying $\left\langle T_{p o l}\right\rangle \eta\left(J_{i}\right)$ with $\pi$ and dividing by the column voltage $V_{D D}$ and an efficacy of $3.9 \mathrm{~lm} / \mathrm{W}$ by multiplying the previous result with $\left\langle\bar{y}_{i}\right\rangle$. As the latter two measures involve the column voltage $V_{D D}$, these measures cover not only the losses in the OLED and polarizer, but also the losses in the TFT and power line. Assuming a polarizer transmission $\left\langle T_{\text {pol }}\right\rangle$ of 0.42 , we obtain for the white OLED an efficacy of $35.2 \mathrm{~cd} / \mathrm{A}$. We also find an efficacy of $9.22 \mathrm{~lm} / \mathrm{W}$ and an efficiency of $2.5 \%$ for the OLED, the TFT and power line combined.

\section{B. Thermal Model}

The temperature distribution across the OLED display is modeled by means of a simplified CAD drawing in FloEFD.

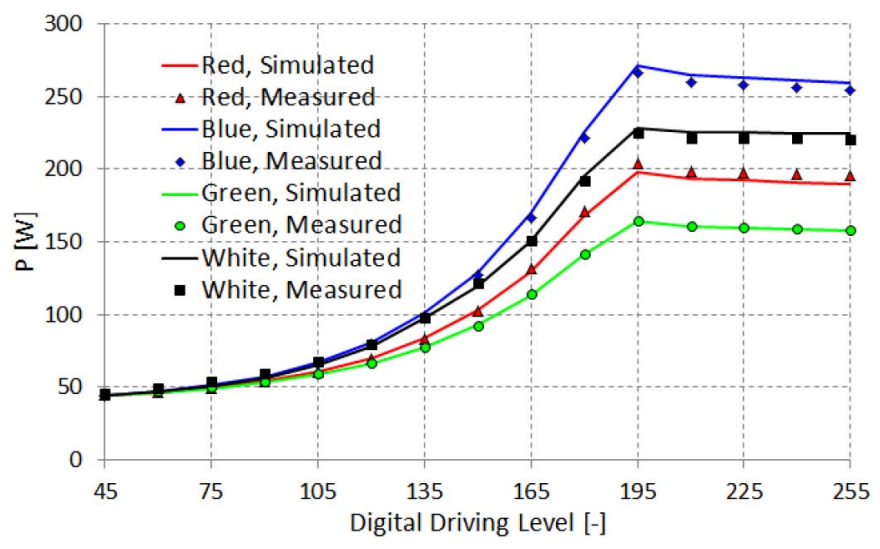

Fig. 14. Simulated (lines) and measured (dots) power consumption $P$ across the display for red (DDL, 0,0$)$, green $(0, \mathrm{DDL}, 0)$, blue $(0,0, \mathrm{DDL})$ and nominal white (DDL,DDL,DDL).

TABLE III

THERMAL MODEL: INPUT AND OUTPUT

\begin{tabular}{|c||c||c||c||c|}
\hline & White & Red & Green & Blue \\
\hline Applied power [W] & 166 & 142.4 & 115.7 & 179.9 \\
\hline Measured max. temperature [C] & 40.49 & 39.15 & 37.44 & 42 \\
\hline Simulated max. temperature [C] & 41.11 & 39.38 & 37.17 & 42.22 \\
\hline
\end{tabular}

The model uses the dimensions of the real display and consists of a surface heat source (representing the OLED layer), sandwiched between two glass layers and integrated into an aluminum frame. In between the aluminum frame and the glass substrate there is an intermediate layer with unknown characteristics. The thermal conductivity of the intermediate layer is tuned such that the simulated temperature gradient across the display corresponds with the measurements. The thermal properties of the materials used in the model are listed in Table II. A schematic of the CAD drawing is shown in Fig. 12 (side-view) and Fig. 13 (front-view and rear-view). The outside condition of the display is air. Heat transportation from 

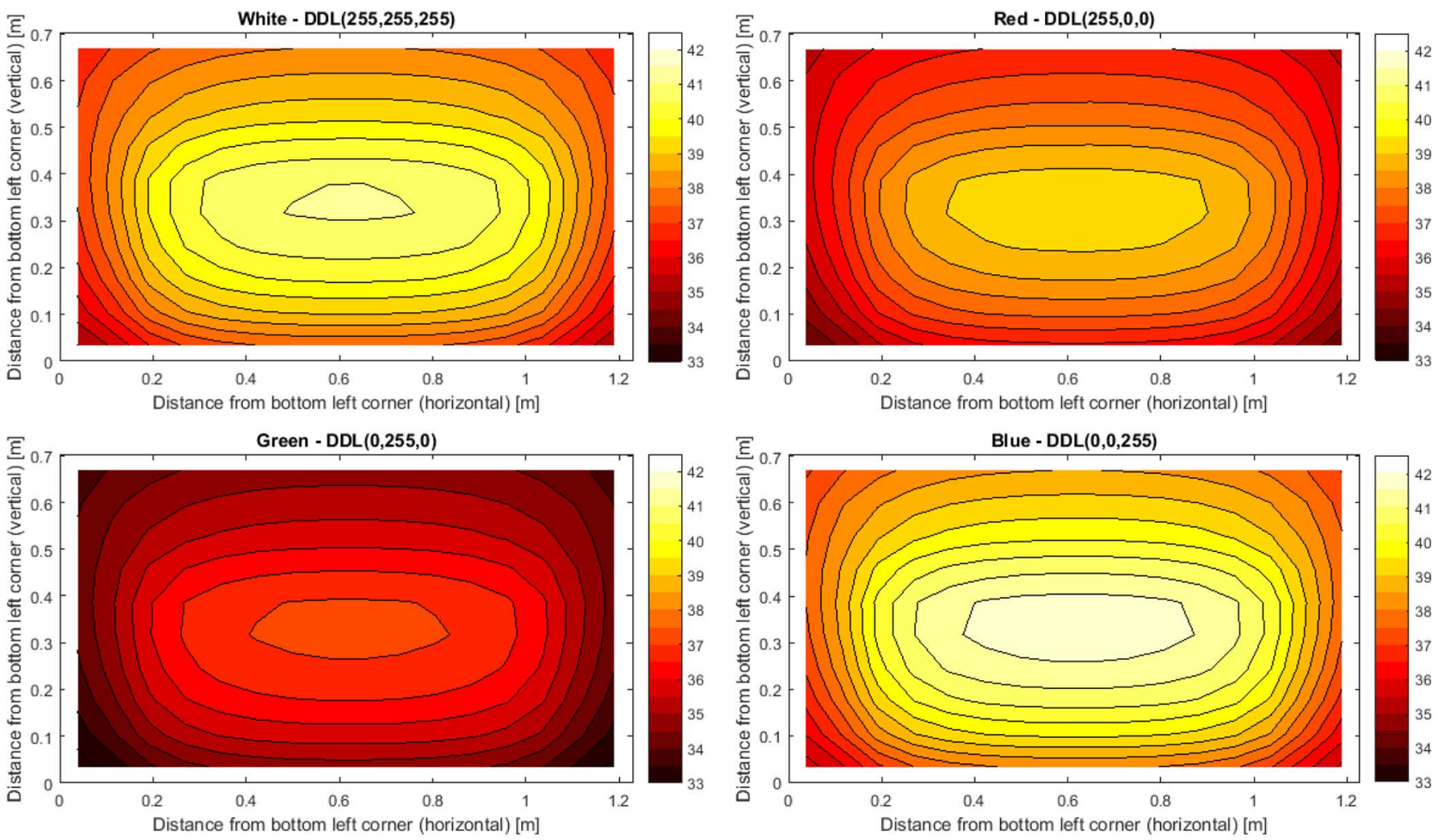

Fig. 15. Simulated steady-state temperature distribution across the OLED display for a uniform full-screen nominal white (top-left), red (top-right), green (bottomleft), and blue patch (bottom-right). The figures show the temperature distribution across the entire display surface. The values on the $\mathrm{x}$ and $\mathrm{y}$-axes correspond to the actual display dimensions. The color bar indicates the temperature in degrees centigrade.

the display to the environment occurs through both thermal radiation and natural convection.

The IR camera image of the back of the display in Fig. 6 (bottom) shows a dark zone in the center part of the display and a bright zone in the outer part of the display. Both zones are seperated by a sharp border. Since the temperature at both sides of the border and close to the border must be approximately the same, the difference in measured radiation can only be attributed to a difference in emissivity of the materials at both sides of the border. Indeed, upon closer inspection of the materials used at the back of the display, it was found that both the center part and the outer part were made up of aluminum but that for the outer part, a plastic foil was put on top of the aluminum. As thermal radiation plays an important role in conditions where there is no forced convection, this difference in material properties had to be implemented in the model to obtain accurate results. The emissivity $\varepsilon$ of the inner part (aluminum) was set to 0.04 . The emissivity of the outer part (plastic foil) was set to 0.94 [cf. Fig. 13(b)].

The thermal model has two inputs: the location and magnitude of the power consumption across the OLED layer and the ambient temperature. The model is capable of calculating the temperature distribution across the display for any given input.

\section{RESUltS AND Discussion}

\section{A. Power Model}

The model described in Section IV-A has been applied to calculate the power consumption of the WRGB OLED display for uniform full-screen patches (all pixels active) at different DDLs. The luminance measurements described in Section III-A and shown in Fig. 2 are used as input to the model. The results are shown in Fig. 14. A good match between power measurements and model output is found.

\section{B. Heat Distribution Model}

To validate the performance of the thermal model, the experiment that was described in Section III-C was mimicked. A uniform power distribution was applied to the OLED layer in the model. The powers that were applied to the OLED layer for each of the four cases can be found in Table III.

The resulting temperature distribution is shown in Fig. 15. The simulated maximum temperature is shown in Table III. The simulated maximum temperature matches well with the measured maximum temperature. Also the temperature distribution matches well with the measurements. At the bottom of the display, a clear difference between measurements and simulations can be observed. The simulations indicate a symmetric temperature distribution in the vertical direction while for the measurements this is not the case. This difference is caused by an additional heat source at the bottom of the display that is not considered in the simulations and that could be attributed to the display drivers. This same effect is also visible on the IR camera image in Fig. 6. Indeed, on the image one observes spots at high temperature at the bottom of the display although none of the OLEDs in the display are active. Other small differences in the shape of the contours could be attributed to slight deviations 
of the locations of the heat sources (different from uniform) or slight deviations in the geometry and properties of the actual materials used in the display. Our aim is to provide a model that is generic for OLED displays and can be used to simulate the temperature across any OLED display. Therefore we limit the components in the model to those that are present in a typical OLED display.

\section{CONCLUSION}

We have measured and analyzed the power consumption of a WRGB OLED display as a function of its luminance. A power model and thermal model have been presented that are respectively capable of calculating the power consumption of a WRGB OLED display as a function of the image content and the temperature distribution across that display. Such models are useful as they allow estimating the power consumption of an OLED display and the temperature distribution across the display as a function of the display characteristics or efficiency. The display's wavelength dependent filter transmission, the efficiency of the white OLED as a function of the current density and the contribution of each of the subpixels in producing the display's nominal white were determined. Analytical expressions for the relationship between consumed power in an OLED and the luminance of the emitted light were derived. Verification of the model output with measurements on a sample 55-in WRGB OLED display with a resolution of $1920 \times 1080$ showed a good match.

\section{REFERENCES}

[1] C. W. Tang and S. A. Van Slyke, "Organic electroluminescent diodes," Appl. Phys. Lett., vol. 51, no. 12, pp. 913-915, Sep. 1987.

[2] N. Odlum, G. Spalla, and N. Van Assche et al., "Preliminary display comparison for dental diagnostic applications," in Proc. SPIE, 2012, vol. 8318 , p. $83181 \mathrm{~S}$.

[3] T. Kimpe and C. Marchessoux, "Important differences between medical displays and normal desktop displays and underlying reasons," in Proc. ADEAC, 2006, pp. 159-162.

[4] S. Berleb, A. G. Mückl, and W. Brütting et al., "Temperature dependent device characteristics of organic light-emitting devices," Synthetic Metals, vol. 111, pp. 341-344, Jun. 2000.

[5] H. Mu, D. Klotzkin, and A. de Silva et al., "Temperature dependence of electron mobility, electroluminescence and photoluminescence of Alq(3) in OLED," J. Phys. D: Appl. Phys., vol. 41, no. 23, Dec. 2008, Art. ID 235109

[6] I. R. de Moraes, S. Scholz, and M. Hermenau et al., "Impact of temperature on the efficiency of organic light emitting diodes," Organic Electron., vol. 26, pp. 158-163, Nov. 2015.

[7] S. Chung, J.-H. Lee, and J. Jeong et al., "Substrate thermal conductivity effect on heat dissipation and lifetime improvement of organic lightemitting diodes," Appl. Phys. Lett., vol. 94, no. 25, Jun. 2009, Art. ID 253302.

[8] M. Ishii and Y. Taga, "Influence of temperature and drive current on degradation mechanisms in organic light-emitting diodes," Appl. Phys. Lett., vol. 80, no. 18, pp. 3430-3432, May 2002.

[9] R. S. Cok, J. Coleman, and F. Leon et al., "Heat distribution in AMOLEDs," J. SID, vol. 13, no. 10, pp. 849-855, Oct. 2005.

[10] J. C. Sturm, W. Wilson, and M. Iodice, "Thermal effects and scaling in organic light-emitting flat-Panel displays," IEEE J. Sel. Top. Quantum. Electron, vol. 4, no. 1, pp. 75-82, 1998.

[11] M.-H. M. Lu, M. Hack, and R. Hewitt et al., "Power consumption and temperature increase in large area active-matrix OLED displays," $J$. Display Technol., vol. 4, no. 1, pp. 47-53, Mar. 2008.
[12] K. J. Bergemann, R. Krasny, and S. R. Forrest, "Thermal properties of organic light-emitting diodes," Organic Electron., vol. 13, no. 9, pp. 1565-1568, Sep. 2012.

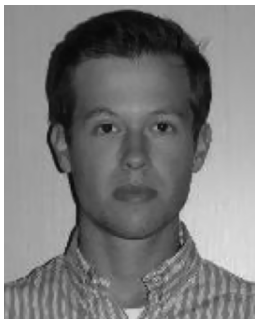

Frédérique Chesterman received the B.S. and M.S degrees in electrical engineering from Ghent University, Belgium, in 2011 and 2013, respectively, where he is currently working toward the Ph.D. degree in photonics engineering in the Electronics and Information Systems department of the Faculty of Engineering Sciences and Architecture. Since 2013, he has been active as a Research Engineer with the Technology and Innovation Group of Barco Healthcare, Kortrijk, Belgium. His research includes topics such as characterization and modeling of OLED displays and development of compensation algorithms, with specific focus on the medical display market.

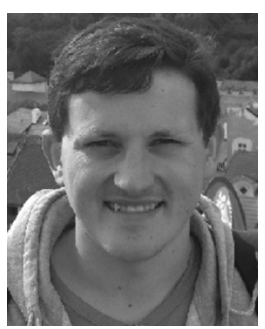

Grigorij Muliuk received the B.S. degree in electronics engineering from Vilnius University, Lithuania, in 2013, and the European M.S. degree in photonics engineering from Ghent University, Belgium, VUB, Belgium and St. Andrews University, Scotland in 2015. His master project research focused on modeling OLED display behavior and development of compensation algorithms. $\mathrm{He}$ is currently working toward the Ph.D. degree at Ghen University in the Photonics Research Group of the department of Information Technology. His current research focuses on integrated photonics and novel ways of heterogeneous integration of photonic components on-a-chip.

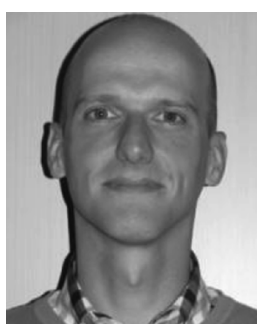

Bastian Piepers received the M.S. degree in electrical engineering from Katholieke Universitei Leuven, Belgium, in 2001. After his graduation he joined as hardware engineer Philips Innovative Applications, developing high end television sets. Afterwards he was with Televic where he first participated to multiple research projects and became later the architect and project lead for the development of nurse call systems. Currently he is the project manager of the Technology and Innovation Group of Barco Healthcare.

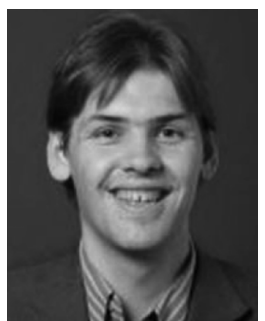

Tom Kimpe received the M.S. degree in computer engineering and Ph.D. degree in electrical engineering from Ghent University, Belgium, in 2001 and 2009 , respectively. His doctoral work focused on the topic of image quality of medical displays. In 2010 he finalized a Master in Business Administration (MBA) at the Vlerick Management School. Since 2001 Tom has been working in Barco's Healthcare Division. He has taken the positions of development engineer, project manager, innovation manager, VP of Technology and Innovation and Chief Technology Officer of Barco's Healthcare Division. Tom is coordinating all research and innovation activities of Barco's Healthcare Division worldwide and is steering an international team of engineers in USA, Belgium and Italy. His main topics of expertise are (medical) display technology, image and signal processing, image quality modelling and human perception, network technology and medical regulatory aspects. Within these fields he is an acknowledged international expert, member of several program committees (e.g., SPIE medical imaging, SID) and reviewer for various journals. Tom also was one of the organizers of the MIPS2015 Medical Imaging Perception Society Conference. 


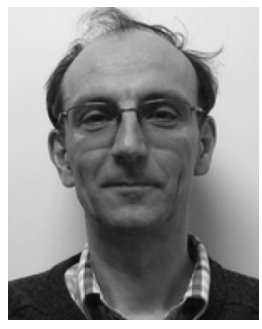

Patrick De Visschere received the degree in Electrical Engineering and $\mathrm{Ph} . \mathrm{D}$. degree in applied sciences from Ghent University in 1976 and 1981, respectively. In 1976 he joined the Department of Electronics and Information Systems (ELIS) of the Ghent University, where he has been working on thin-film solar cells. From 1984 until 2000 he was affiliated with IMEC, and did research on thin-film electroluminescence. In 1993, he became part-time, and in 2000 a full time Associate Professor in physical electronics and visualisation technology at the Ghent University. His main interests are in physical electronics and optics, electromagnetism and ray-tracing.

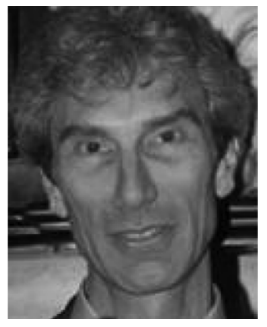

Kristiaan Neyts is a Full Research Professor with Ghent University in the Electronics and Information Systems department of the Faculty of Engineering Sciences and Architecture. He received the Ph.D. degree from Ghent University in 1992 on thin film electroluminescence and made a post-doc at UC Berkeley in 1997-1998. Now he is heading the Liquid Crystals and Photonics group that is conducting research in the fields of liquid crystals, OLEDs, electrophoresis and microscopy. This group of 20 researchers has expertise in device technology, numerical simulations and electro-optical characterization of photonic components. Since 2004, he has been the promoter of 18 completed PhDs. 